\title{
Are there compact heavy four-quark bound states?
}

\author{
J. Vijande, ${ }^{1}$ E. Weissman, ${ }^{2}$ A. Valcarce, ${ }^{3}$ and N. Barnea ${ }^{2,4}$ \\ ${ }^{1}$ Departamento de Física Teórica e IFIC, Universidad de Valencia-CSIC, E-46100 Burjassot, Valencia, Spain \\ ${ }^{2}$ The Racah Institute of Physics, The Hebrew University, 91904, Jerusalem, Israel \\ ${ }^{3}$ Departamento de Física Fundamental, Universidad de Salamanca, E-37008 Salamanca, Spain \\ ${ }^{4}$ Institute for Nuclear Theory, University of Washington, Seattle, Washington 98195, USA \\ (Received 30 May 2007; revised manuscript received 28 September 2007; published 28 November 2007)
}

We present an exact method to study four-quark systems based on the hyperspherical harmonics formalism. We apply it to several physical systems of interest containing two heavy and two light quarks using different quark-quark potentials. Our conclusions mark the boundaries for the possible existence of compact, nonmolecular, four-quark bound states. While $Q Q \bar{n} \bar{n}$ states may be stable in nature, the stability of $Q \bar{Q} n \bar{n}$ states would imply the existence of quark correlations not taken into account by simple quark dynamical models.

The discoveries on several fronts [1], of unusual charmonium states like $X(3872)$ and $Y(4260)$ and open-charm mesons with unexpected masses like $D_{s J}^{*}(2317)$ and $D_{0}^{*}(2308)$, have reinvigorated the study of the hadron spectra. Their anomalous nature has triggered several interpretations, among them, the existence of four-quark states or meson-meson molecules. This challenging situation resembles the long-standing problem of the light-scalar mesons, where it has been suggested that some resonances may not be ordinary $q \bar{q}$ states, though there is little agreement on what they actually are [2]. In this case, four-quark states have been justified to coexist with $q \bar{q}$ states because they can couple to $J^{P C}=0^{++}$without orbital excitation [3].

Any debate on the possible multiquark structure of meson resonances should be based on our capability to find an exact solution of the four-body problem [4]. Theoretical predictions often differ because of the approximation method used. A powerful tool to solve a fewparticle system is to expand the trial wave function in terms of hyperspherical harmonics $(\mathrm{HH})$ basis functions. This method has been proven to be rather powerful to solve the nuclear [5] four-body problem. In this work we use a generalization of the $\mathrm{HH}$ formalism to study four-quark systems in an exact way. There are two basic difficulties for constructing $\mathrm{HH}$ functions of proper symmetry for a system of identical particles: first, the simultaneous treatment of particles and antiparticles, and second the additional color and flavor degrees of freedom. The method will be tested by comparing with the existing results based on different approximate solutions, thus establishing the validity of such approximations. Because of their actual interest and having in mind that systems with unequal masses are more promising to be bound [4], we will center on the study of $Q Q \bar{n} \bar{n}$ and $Q \bar{Q} n \bar{n}$ states ( $n$ stands for a light quark and $Q$ for a heavy one). We will analyze the possible existence of compact four-quark bound states using two standard quark-quark interactions, a Bhadurilike potential $(\mathrm{BCN})[6]$ and a constituent quark model considering boson exchanges (CQC) [7]. Both interactions give a reasonable description of the meson and the baryon spectroscopy, a thoughtful requirement considering that in the tetraquarks $q q$ and $q \bar{q}$ interactions will contribute.

Within the $\mathrm{HH}$ expansion, the four-quark wave function can be written as a sum of outer products of color, isospin, spin, and configuration terms,

$$
\left.\left.\left|\phi_{\mathrm{CISR}}\right\rangle=\mid \text { Color }\right\rangle \mid \text { Isospin }\right\rangle[|\operatorname{Spin}\rangle \otimes|R\rangle]^{J M},
$$

such that the four-quark state is a color singlet with welldefined parity, isospin, and total angular momentum. In the following, we shall assume that particles 1 and 2 are the $Q$-quarks and particles 3 and 4 are the $n$-quarks. In the $Q Q \bar{n} \bar{n}$ case particles 1 and 2 are identical, and so are 3 and 4. Consequently, the Pauli principle leads to the following conditions:

$$
\hat{P}_{12}\left|\phi_{\mathrm{CISR}}\right\rangle=\hat{P}_{34}\left|\phi_{\mathrm{CISR}}\right\rangle=-\left|\phi_{\mathrm{CISR}}\right\rangle,
$$

$\hat{P}_{i j}$ being the permutation operator of particles $i$ and $j$.

Coupling the color states of two quarks (antiquarks) can yield two possible representations, the symmetric 6dimensional, $6(\overline{6})$, and the antisymmetric 3-dimensional, $\overline{3}$ (3). Coupling the color states of the quark pair with that of the antiquark pair must yield a color singlet. Thus, there are only two possible color states for a $Q Q \bar{q} \bar{q}$ system [8],

$$
\mid \text { Color }\rangle=\left\{\left|\overline{3}_{12} 3_{34}\right\rangle,\left|6_{12} \overline{6}_{34}\right\rangle\right\} \text {. }
$$

These states have well-defined symmetry under permutations, Eq. (2). The spin states with such symmetry can be obtained in the following way:

$$
\mid \text { Spin }\rangle=\left|\left(\left(s_{1}, s_{2}\right) S_{12},\left(s_{3}, s_{4}\right) S_{34}\right) S\right\rangle=\left|\left(S_{12} S_{34}\right) S\right\rangle .
$$

The same holds for the isospin, |Isospin $\rangle=\left|\left(i_{3}, i_{4}\right) I_{34}\right\rangle$, which applies only to the $n$-quarks, thus $I=I_{34}$.

As mentioned above, we use the HH expansion to describe the spatial part of the wave function. We choose for 
convenience the $H$-type Jacobi coordinates,

$\boldsymbol{\eta}_{1}=\mu_{1,2}\left(\boldsymbol{r}_{2}-\boldsymbol{r}_{1}\right)$,

$\boldsymbol{\eta}_{2}=\mu_{12,34}\left(\frac{m_{3} \boldsymbol{r}_{3}+m_{4} \boldsymbol{r}_{4}}{m_{34}}-\frac{m_{1} \boldsymbol{r}_{1}+m_{2} \boldsymbol{r}_{2}}{m_{12}}\right)$,

$\boldsymbol{\eta}_{3}=\mu_{3,4}\left(\boldsymbol{r}_{4}-\boldsymbol{r}_{3}\right)$,

where $m_{i j}=m_{i}+m_{j}, \mu_{i, j}=\sqrt{m_{i} m_{j} / m_{i j}}$, and $m_{1234}=$ $m_{1}+m_{2}+m_{3}+m_{4}$. Using these vectors, it is easy to obtain basis functions that have well-defined symmetry under permutations of the pairs (12) and (34). In the $\mathrm{HH}$ formalism the three Jacobi vectors are transformed into a single length variable, $\rho=\sqrt{\eta_{1}^{2}+\eta_{2}^{2}+\eta_{3}^{2}}$, and 8-angular variables, $\Omega$, that represent the location on the 8dimensional sphere. The spatial basis states are given by

$$
\langle\rho \Omega \mid R\rangle=U_{n}(\rho) \mathcal{Y}_{[K]}(\Omega),
$$

where $\mathcal{Y}_{[K]}$ are the $\mathrm{HH}$ functions, and $[K] \equiv$ $\left\{K K_{12} L M_{L} L_{12} \ell_{3} \ell_{2} \ell_{1}\right\}$. The quantum number $K$ is the grand angular momentum, $L M_{L}$ are the usual orbital angular momentum quantum numbers, and $\ell_{i}$ is the angular momentum associated with the Jacobi vector $\eta_{i}$. The quantum numbers $K_{12}, L_{12}$ correspond to the intermediate coupling of $\eta_{1}$ and $\eta_{2}$. The Laguerre functions are used as the hyper-radial basis functions $U_{n}(\rho)$.

The Pauli principle, Eq. (2), leads to the following restrictions on the allowed combinations of basis states:

(i) $(-1)^{S_{12}+\ell_{1}}=+1, \quad(-1)^{S_{34}+I+\ell_{3}}=-1$ for the $\left|6_{12} \overline{6}_{34}\right\rangle$ color state,

(ii) $(-1)^{S_{12}+\ell_{1}}=-1, \quad(-1)^{S_{34}+I+\ell_{3}}=+1$ for the $\left|\overline{3}_{12} 3_{34}\right\rangle$ state.

In the $Q \bar{Q} n \bar{n}$ case, particle 2 is the antiparticle of particle 1 , and particle 4 is the antiparticle of particle 3. Assuming that $C$-parity is a good symmetry of QCD, we can regard quarks and antiquarks as identical particles and impose the symmetry condition, Eq. (2), on the $Q \bar{Q} n \bar{n}$ system as well. Coupling the color states of a quark and an antiquark can yield two possible representations: the singlet and the octet. These representations should be combined in the following way [8] $\left\{\left|1_{12} 1_{34}\right\rangle,\left|8_{12}, 8_{34}\right\rangle\right\}$ to yield a total color singlet state. However, these states have not definite symmetry under particle permutations (12) and (34). To construct symmetrized states for the $Q \bar{Q}$ pair, we consider the following combinations:

$$
\left|C_{12}^{\Gamma_{12}}\right\rangle=\frac{1}{\sqrt{2}}\left(\left|C_{12}\right\rangle+\Gamma_{12}\left|C_{21}\right\rangle\right),
$$

where $C_{12}=\left\{1_{12}, 8_{12}\right\}$, and $\Gamma_{12}=+1$ for a symmetric combination and -1 for an antisymmetric one. For light quarks the color and isospin states should be combined together to form states with well-defined symmetry. For $I_{z}=0$, for instance, these states take the form

$$
\begin{aligned}
\left|\left(C_{34} I_{34}\right)^{\Gamma_{34}}\right\rangle= & +\frac{1}{2}\left[\left|C_{34}\right\rangle(|u \bar{u}\rangle \pm|d \bar{d}\rangle)+\Gamma_{34}\left|C_{43}\right\rangle\right. \\
& \times(|\bar{u} u\rangle \pm|\bar{d} d\rangle)],
\end{aligned}
$$

where the plus sign stands for the $I_{34}=0$ state and the minus sign for the $I_{34}=1$ state. As before, $C_{34}$ stands for either the singlet or the octet representations. The total color-isospin states, $\left|C_{12}^{\Gamma_{12}}\left(C_{34} I_{34}\right)^{\Gamma_{34}}\right\rangle$ are not only good symmetry states, but also good $C$-parity states with $C=$ $\Gamma_{12} \Gamma_{34}$. Imposing the Pauli principle for the $Q \bar{Q} n \bar{n}$ system we get the following restrictions: $\Gamma_{12}(-1)^{S_{12}+\ell_{1}}=+1$, $\Gamma_{34}(-1)^{S_{34}+\ell_{3}}=+1$, on the basis states.

Assuming nonrelativistic quantum mechanics, we solve the four-body Schrödinger equation using the basis states described above. The grand angular momentum $K$ is the main quantum number in our expansion and the calculation is truncated at some $K$ value. As mentioned above, for our study we will use two standard quark potential models providing a reasonable description of the hadron spectra. In the following we draw the basic properties of the interacting potentials.

The BCN model was proposed in the early 1980's by Bhaduri et al. in an attempt to obtain a unified description of meson and baryon spectroscopy [6]. It was later applied to study the baryon spectra [9] and four-quark ( $q q \bar{q} \bar{q})$ systems [10]. The model retains the most important terms of the one-gluon exchange interaction proposed by de Rújula et al. [11], namely, Coulomb and spin-spin terms, and a linear confining potential, having the form

$$
\begin{aligned}
V\left(\vec{r}_{i j}\right)= & -\frac{3}{16}\left(\vec{\lambda}_{i}^{c} \cdot \vec{\lambda}_{j}^{c}\right)\left(\frac{r_{i j}}{a^{2}}-\frac{\kappa}{r_{i j}}-D\right. \\
& \left.+\frac{\kappa}{m_{i} m_{j}} \frac{e^{-r_{i j} / r_{0}}}{r_{i j} r_{0}^{2}}\left(\vec{\sigma}_{i} \cdot \vec{\sigma}_{j}\right)\right),
\end{aligned}
$$

where $\vec{\sigma}_{i}$ are the Pauli matrices and $\vec{\lambda}_{i}^{c}$ are the $S U(3)$ color matrices. The parameters $\kappa=102.67 \mathrm{MeVfm}, D=$ $913.5 \mathrm{MeV}, \quad a=0.0326 \mathrm{MeV}^{-1 / 2} \mathrm{fm}^{1 / 2}, \quad r_{0}=2.2 \mathrm{fm}$, $m_{u, d}=337 \mathrm{MeV}$, and $m_{c}=1870 \mathrm{MeV}$ are taken from Ref. [10].

The CQC model was proposed in the early 1990's in an attempt to obtain a simultaneous description of the nucleon-nucleon interaction and the baryon spectra [12]. It was later generalized to all flavor sectors giving a reasonable description of the meson [7] and baryon spectra [13]. The possible existence of four-quark states within this model has also been addressed $[14,15]$.

The model is based on the assumption that the lightquark constituent mass appears because of the spontaneous breaking of the original $S U(3)_{L} \otimes S U(3)_{R}$ chiral symmetry at some momentum scale. In this domain of momenta, quarks interact through Goldstone boson exchange potentials. QCD perturbative effects are taken into account through the one-gluon-exchange (OGE) potential as the one used in the BCN model. Finally, it incorporates confinement as dictated by unquenched lattice calculations 
predicting, for heavy quarks, a screening effect on the linearly dependent interquark potential when increasing the interquark distance [16].

The model parameters have been taken from Ref. [7] with the exception of the OGE regularization parameter. This parameter, taking into account the size of the system, was fitted for four-quark states in the description of the light-scalar sector [14], being $\hat{r}_{0}=0.18 \mathrm{fm}$ for mesons and $\hat{r}_{0}=0.38 \mathrm{fm}$ for four-quark systems. Let us also notice that the CQC model contains an interaction generating flavor mixing between $n \bar{n}$ and $s \bar{s}$ components. It allows one to exactly reproduce the masses of the $\eta$ and $\eta^{\prime}$ mesons. In the four-quark case this contribution would mix isospin zero $Q \bar{Q} n \bar{n}$ and $Q \bar{Q} s \bar{s}$ components. Such contributions were explicitly evaluated in the variational approach of Ref. [14] for the light isocalar tetraquarks, giving a negligible effect. In order to make a proper comparison between thresholds and four-quark states, we have recalculated the meson spectra of Ref. [7] with the same $\hat{r}_{0}$ value and interaction used in the four-quark calculation, neglecting therefore the flavor-mixing terms. Explicit expressions of the interacting potentials and a more detailed discussion of the model can be found in Ref. [7].

Let us first analyze the numerical capability of the designed method to capture the properties of the four-quark systems. In Table I we present the results for different $L=$ 0 spin-isospin $c c \bar{n} \bar{n}$ states calculated with the CQC model. We quote in the first column the results obtained within a variational calculation using Gaussian trial wave functions only with quadratic terms in the Jacobi coordinates [17]. This approximation would correspond in our formalism to set $\ell_{i}=0$ for the three Jacobi vectors. These results are given in the second column, reproducing exactly the variational results. The validity of this approximation can be judged by looking at the last column where we give the exact $\mathrm{HH}$ results, truncated at $K=24$. In some cases the difference between the $\ell_{i}=0$ approximation and the true ground state can be as large as $200 \mathrm{MeV}$. We have also reproduced the calculation of the $(S, I)=(1,0) L=0$ $c c \bar{n} \bar{n}$ state of Refs. [10,18] using the BCN model. For $K=$ 24 we have obtained an energy of $3899.7 \mathrm{MeV}$ as compared to 3904.7 MeV of Ref. [18] and 3931.0 MeV of Ref. [10]. Reference [18] designed a powerful method, similar to the stochastic variational approach [19], to study this particular system. Although their results are not fully converged, the close agreement gives confidence on both calculations. The

TABLE I. Energy (MeV) of $L=0 c c \bar{n} \bar{n}$ states.

\begin{tabular}{cccc}
\hline \hline (S,I) & Ref. [17] & $\mathrm{HH}\left(\ell_{i}=0\right)$ & $\mathrm{HH}$ \\
\hline$(0,1)$ & 4155 & 4154 & 3911 \\
$(1,0)$ & 3927 & 3926 & 3860 \\
$(1,1)$ & 4176 & 4175 & 3975 \\
$(2,1)$ & 4195 & 4193 & 4031 \\
\hline \hline
\end{tabular}

results of Ref. [10] were obtained using diagonalization in harmonic oscillator (HO) basis up to $N=8$. The quality of this last procedure can be judged by looking at Table II where we compare, for different $c \bar{c} n \bar{n}$ states, the results of [10] to the HH results with $K \leq 8$ and to the converged $\mathrm{HH}$ results obtained with $K$ restricted by our computational capability, i.e. $K=22$ or $K=26$. As can be seen, the results with the basis truncated at $K=8$ are similar to the HO results, but rather far from the converged ones.

In spite of the shortcomings of the methods used to study four-quark systems, in the past, many four-quark bound states have been suggested. To analyze their stability against dissociation, the parity and the total angular momentum must be preserved. Additionally, $C$-parity is a good quantum number for $c \bar{c} n \bar{n}$ and the Pauli principle must be fulfilled in the $c c \bar{n} \bar{n}$ case. The thresholds can be evaluated by adding the meson masses of the dissociation channel. A four-quark state will be stable under strong interaction, and therefore narrow, if its mass lies below all allowed two-meson thresholds. Sometimes, the results of four-quark calculations have been directly compared to the experimental thresholds. In this case, one could misidentify scattering wave functions as bound states. When they are referred to the threshold within the same model, we will see how the theoretical predictions do not imply an abundance of multiquark states in the data.

Let us make a brief comment on the convergence of the $\mathrm{HH}$ expansion. In some cases the convergence is slow, as can be seen by comparing Tables III and IV. This is a consequence of the $\mathrm{HH}$ formalism being better suited to treat with bound states, and most of the four-quark states one deals with are above the corresponding two-meson threshold, as can be seen in Table III. Because of this slowness, our calculation cannot definitively exclude large molecular objects (sizes of the order of 1.5-2 fm) with binding energies smaller than $1-2 \mathrm{MeV}$ induced by longrange interactions like, for instance, the one-pion exchange tail [20].

Once the method has been designed, tested, and its accuracy established, we concentrate on a hot subject: the $c \bar{c} n \bar{n}$ system as a potential structure for the $X(3872)$. To make the physics clear we will compare with the $c c \bar{n} \bar{n}$ system. In particular, we focus on the $J^{P C}=1^{++} c \bar{c} n \bar{n}$ and $J^{P}=1^{+} c c \bar{n} \bar{n}$ quantum numbers to illustrate their similitude and differences. A complete study of all the quantum numbers will be reported elsewhere. The results are shown in Tables III and IV up to the maximum value of $K$ within our computational capabilities. Since we are using a complete set of $\mathrm{HH}$ basis, all possible configurations are con-

TABLE II. Energy $(\mathrm{MeV})$ of $L=0 c \bar{c} n \bar{n}$ states.

\begin{tabular}{lccc}
\hline \hline$J^{P}$ & Ref. [10] & $\mathrm{HH}(K=8)$ & $\mathrm{HH}\left(K_{\max }\right)$ \\
\hline $0^{+}$ & 3409 & 3380 & $3249(26)$ \\
$1^{+}$ & 3468 & 3436 & $3319(22)$ \\
\hline \hline
\end{tabular}


TABLE III. Energy (MeV) and probability of the different color components as a function of $K$ for the $c \bar{c} n \bar{n} J^{P C}=1^{++}$ state both for CQC and BCN models. The last rows indicate the lowest theoretical two-meson thresholds. The notation $\left.\right|_{S}\left(\left.\right|_{P}\right)$ stands for relative $S$-wave ( $P$-wave).

\begin{tabular}{lcccccc}
\hline \hline & \multicolumn{3}{c}{$\mathrm{CQC}$} & & & $\mathrm{BCN}$ \\
$K$ & $\mathrm{E}$ & $P_{11}$ & $P_{88}$ & $\mathrm{E}$ & $P_{11}$ & $P_{88}$ \\
\hline 0 & 4141 & 1.0000 & 0.0000 & 4196 & 1.0000 & 0.0000 \\
2 & 3985 & 0.9822 & 0.0178 & 4053 & 0.9462 & 0.0538 \\
4 & 3911 & 0.9789 & 0.0211 & 3994 & 0.9233 & 0.0767 \\
6 & 3870 & 0.9834 & 0.0166 & 3963 & 0.9236 & 0.0764 \\
8 & 3845 & 0.9871 & 0.0129 & 3944 & 0.9303 & 0.0697 \\
10 & 3827 & 0.9905 & 0.0095 & 3932 & 0.9426 & 0.0574 \\
12 & 3814 & 0.9926 & 0.0074 & 3920 & 0.9927 & 0.0073 \\
14 & 3805 & 0.9943 & 0.0057 & 3887 & 0.9990 & 0.0010 \\
16 & 3797 & 0.9954 & 0.0046 & 3861 & 0.9994 & 0.0006 \\
18 & 3791 & 0.9962 & 0.0038 & 3840 & 0.9995 & 0.0005 \\
20 & 3786 & 0.9968 & 0.0032 & 3822 & 0.9996 & 0.0004 \\
22 & $\cdots$ & $\cdots$ & $\cdots$ & 3808 & 0.9997 & 0.0003 \\
\hline$J /\left.\psi \omega\right|_{S}$ & 3745 & 1 & 0 & 3874 & 1 & 0 \\
$\left.\chi_{C J} \eta\right|_{P}$ & 4281 & 1 & 0 & 3655 & 1 & 0 \\
\hline \hline
\end{tabular}

sidered in both cases. For the $c \bar{c} n \bar{n}$ system, independently of the quark-quark interaction, the system evolves to a well separated two-meson state. This is clearly seen in the energy, approaching the corresponding two free-meson threshold, but also in the probabilities of the different color components of the wave function and in the radius. We denote by $P_{11}\left(P_{88}\right)$ the probability of a singlet-singlet (octet-octet) color component in the $(c \bar{c})(n \bar{n})$ [or $(c \bar{n}) \times$ $(c \bar{n})$ ] coupling. We observe how the system evolves to two singlet color mesons, whose separation increases with $K$, dashed line in Fig. 1. Comparing the theoretical predictions with the experimental threshold, $M_{J /\left.\psi \omega\right|_{S}}=3879.57 \pm$

TABLE IV. Same as Table III for the $c c \bar{n} \bar{n} J^{P}=1^{+}$state.

\begin{tabular}{lcccccc}
\hline \hline & & $\mathrm{CQC}$ & & & $\mathrm{BCN}$ & \\
$K$ & $\mathrm{E}$ & $P_{11}$ & $P_{88}$ & $\mathrm{E}$ & $P_{11}$ & $P_{88}$ \\
\hline 0 & 4109 & 0.3351 & 0.6649 & 4100 & 0.3446 & 0.6554 \\
2 & 3990 & 0.3483 & 0.6517 & 3999 & 0.3744 & 0.6256 \\
4 & 3931 & 0.3577 & 0.6423 & 3954 & 0.3981 & 0.6019 \\
6 & 3903 & 0.3641 & 0.6359 & 3933 & 0.4170 & 0.5830 \\
8 & 3887 & 0.3681 & 0.6319 & 3921 & 0.4302 & 0.5698 \\
10 & 3878 & 0.3705 & 0.6295 & 3914 & 0.4403 & 0.5597 \\
12 & 3872 & 0.3720 & 0.6280 & 3910 & 0.4478 & 0.5522 \\
14 & 3868 & 0.3730 & 0.6270 & 3907 & 0.4536 & 0.5464 \\
16 & 3866 & 0.3737 & 0.6263 & 3904 & 0.4581 & 0.5419 \\
18 & 3864 & 0.3741 & 0.6259 & 3903 & 0.4618 & 0.5382 \\
20 & 3862 & $\cdots$ & $\cdots$ & 3901 & 0.4647 & 0.5353 \\
22 & 3861 & $\cdots$ & $\cdots$ & 3900 & $\cdots$ & $\cdots$ \\
24 & 3861 & $\cdots$ & $\cdots$ & 3900 & $\cdots$ & $\cdots$ \\
\hline$\left.D D^{*}\right|_{S}$ & 3937 & 1 & 0 & 3906 & 1 & 0 \\
\hline \hline
\end{tabular}

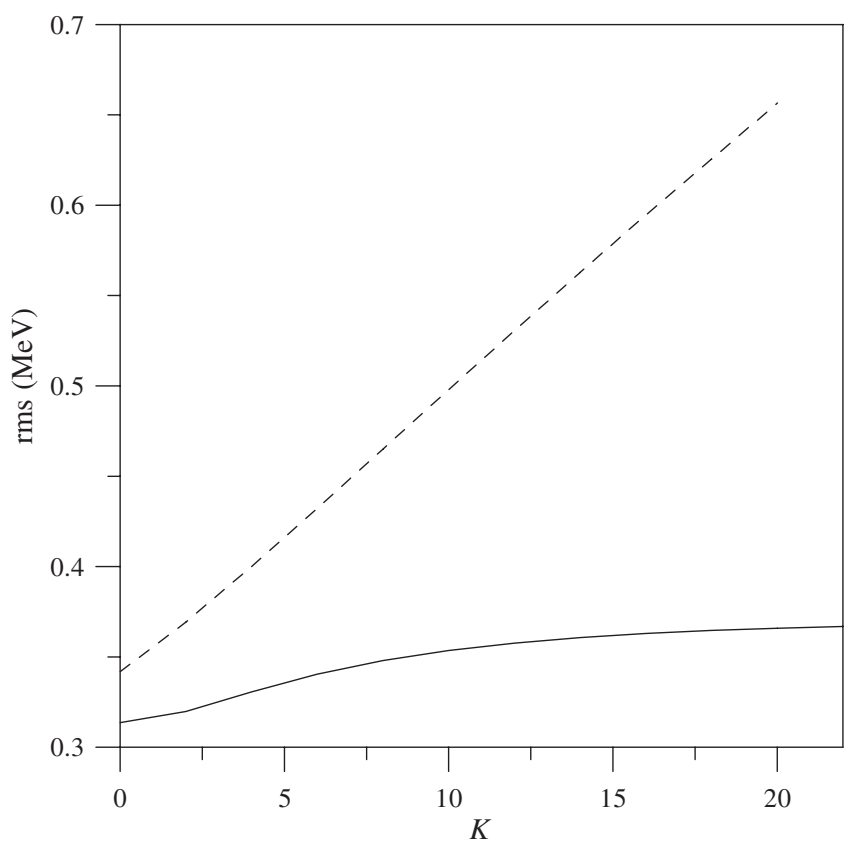

FIG. 1. Evolution with $K$ of the root-mean square radius (rms) of the $c \bar{c} n \bar{n} J^{P C}=1^{++}$state (dashed line) and the $c c \bar{n} \bar{n} J^{P}=$ $1^{+}$state (solid line) for the CQC model.

$0.13 \mathrm{MeV}$, one could be tempted to claim for the existence of a bound state. However, the experimental threshold is not reproduced by the effective Hamiltonians. In fact, in the BCN model the sum of the masses of the two mesons $J / \psi \omega$ is even larger than that of $\chi_{c J} \eta$, leading to a completely different threshold for the $1^{++}$system. Thus, in any manner one can claim for the existence of a bound state. Similar conclusions are drawn for all quantum numbers of this system.

A completely different behavior is observed in Table IV. Here, the energy is quickly stabilized below the theoretical threshold. Besides, the radius is also stable, solid line in Fig. 1, and it is smaller than the sum of the radius of the two-meson threshold. We obtain $r_{4 q}=0.37 \mathrm{fm}$ compared to $r_{M_{1}}+r_{M_{2}}=0.44 \mathrm{fm}$. We also notice a different solution for the probability of the color components. However, one should not directly conclude the presence of octetoctet components, because the octet-octet color component in the $\left(c_{1} \bar{n}_{3}\right)\left(c_{2} \bar{n}_{4}\right)$ basis can be reexpressed as a singletsinglet color component in the $\left(c_{1} \bar{n}_{4}\right)\left(c_{2} \bar{n}_{3}\right)$ coupling, being the same physical system due to the identity of the two quarks and the two antiquarks. Although in the BCN model the system is slightly bound, the structure of the bound state is manifest for low values of $K$, leading one to conclude that the state could hardly be destroyed by small nonconsidered effects as could be, for example, relativity. The actual interest and the capability of some experiments [21] to detect double charm states makes this prediction a primary objective to help in the understanding of QCD dynamics. 
It is thus important to realize that a bound state should be pursued not only by looking at the energy, but also with a careful analysis of the radius and color probabilities. Besides, one should compare results within the same calculating framework, unless other effects, as discussed below, are considered. This detailed analysis allows us to distinguish between compact states and meson-meson molecules [3] and it does consider the contribution of all meson-meson channels to a particular set $J^{P C}$ of quantum numbers [20]. Inherent to our discussion is a much richer decay spectrum of compact states due to the presence of octet-octet color components in their wave function.

Let us notice that there is an important difference between the two physical systems studied. While for the $c \bar{c} n \bar{n}$ there are two allowed physical decay channels, $(c \bar{c})(n \bar{n})$ and $(c \bar{n})(\bar{c} n)$, for the $c c \bar{n} \bar{n}$ only one physical system contains the possible final states, $(c \bar{n})(c \bar{n})$. This has important consequences if both systems (two- and four-quark states) are described within the same twobody Hamiltonian, the $c \bar{c} n \bar{n}$ will hardly present bound states, because the system will reorder itself to become the lightest two-meson state, either $(c \bar{c})(n \bar{n})$ or $(c \bar{n})(\bar{c} n)$. In other words, if the attraction is provided by the interaction between particles $i$ and $j$, it does also contribute to the asymptotic two-meson state. This does not happen for the $c c \bar{n} \bar{n}$ if the interaction between, for example, the two quarks is strongly attractive. In this case there is no asymptotic two-meson state including such attraction, and therefore the system will bind.

Therefore, our conclusions can be made more general. If we have an $\mathrm{N}$-quark system described by two-body interactions in such a way that there exists a subset of quarks that cannot make up a physical subsystem, then one may expect the existence of $N$-quark bound states by means of central two-body potentials. If this is not true, one will hardly find $N$-quark bound states [22]. For the particular case of the tetraquarks, this conclusion is exact if the confinement is described by the first $S U$ (3) Casimir operator, because when the system is split into two-mesons the confining contribution from the two isolated mesons is the same as in the four-quark system. The contribution of three-body color forces [23] would interfere in the simple comparison of the asymptotic and the compact states. Another possibility in the same line would be a modification of the Hilbert space. If for some reason particular components of the four-quark system (diquarks) would be favored against others, the system could be compact [24]. Lattice QCD calculations [25] confirm the phenomenological expectation that QCD dynamics favors the formation of good diquarks [3], i.e., in the scalar positive parity channel. However, they are large objects whose relevance to hadron structure is still under study. All these alternatives will allow one to manage the four-quark system without affecting the threshold and thus they may allow one to generate any solution.

Let us finally note that in Ref. [26] the stability of the $Q Q \bar{n} \bar{n}$ and $Q \bar{Q} n \bar{n}$ systems has been analyzed in a simple string model considering only a multiquark confining interaction given by the minimum of a flip-flop or a butterfly potential. The ground state of systems made of two quarks and two antiquarks of equal masses was found to be below the dissociation threshold. While for the flavor exotic $Q Q \bar{n} \bar{n}$ the binding increases when increasing the mass ratio $m_{Q} / m_{q}$, for the cryptoexotic $Q \bar{Q} n \bar{n}$ the effect of symmetry breaking is the opposite, the system being unbound whenever $m_{Q} / m_{q}>1$. Although more realistic calculations are needed before establishing a definitive conclusion, the findings of Ref. [26] strengthened our results.

We thank Dr. L. Sommovigo for a careful reading of the manuscript. This work has been partially funded by Ministerio de Ciencia y Tecnología under Contract No. FPA2007-65748, and by Junta de Castilla y León under Contract No. SA016A07.
[1] W.-M. Yao et al., J. Phys. G 33, 1 (2006).

[2] C. Amsler and N. A. Tornqvist, Phys. Rep. 389, 61 (2004).

[3] R. L. Jaffe, Phys. Rep. 409, 1 (2005); arXiv:hep-ph/ 0701038.

[4] J. P. Ader, J.-M. Richard, and P. Taxil, Phys. Rev. D 25, 2370 (1982); J. L. Ballot and J.-M. Richard, Phys. Lett. 123B, 449 (1983); H. J. Lipkin, Phys. Lett. B 172, 242 (1986); L. Heller and J. A. Tjon, Phys. Rev. D 32, 755 (1985); 35, 969 (1987).

[5] M. Viviani, A. Kievsky, and S. Rosati, Few-Body Syst. 18, 25 (1995).

[6] R. K. Bhaduri, L.E. Cohler, and Y. Nogami, Nuovo Cimento A 65, 376 (1981).
[7] J. Vijande, F. Fernández, and A. Valcarce, J. Phys. G 31, 481 (2005).

[8] R. L. Jaffe, Phys. Rev. D 15, 267 (1977); 15, 281 (1977).

[9] B. Silvestre-Brac and C. Gignoux, Phys. Rev. D 32, 743 (1985).

[10] B. Silvestre-Brac and C. Semay, Z. Phys. C 57, 273 (1993).

[11] A. de Rújula, H. Georgi, and S. L. Glashow, Phys. Rev. D 12, 147 (1975).

[12] A. Valcarce, H. Garcilazo, F. Fernández, and P. González, Rep. Prog. Phys. 68, 965 (2005).

[13] A. Valcarce, H. Garcilazo, and J. Vijande, Phys. Rev. C 72, 025206 (2005). 
[14] J. Vijande, A. Valcarce, F. Fernández, and B. SilvestreBrac, Phys. Rev. D 72, 034025 (2005).

[15] N. Barnea, J. Vijande, and A. Valcarce, Phys. Rev. D 73, 054004 (2006); J. Vijande, A. Valcarce, and K. Tsushima, Phys. Rev. D 74, 054018 (2006).

[16] G.S. Bali, Phys. Rep. 343, 1 (2001), and references therein.

[17] J. Vijande et al., Eur. Phys. J. A 19, 383 (2004).

[18] D. Janc and M. Rosina, Few-Body Syst. 35, 175 (2004).

[19] K. Varga and Y. Suzuki, Phys. Rev. C 52, 2885 (1995).
[20] N. A. Törnqvist, Phys. Lett. B 590, 209 (2004).

[21] A. Ocherashvili et al. (SELEX Collaboration), Phys. Lett. B 628, 18 (2005).

[22] H. J. Lipkin, Phys. Lett. 58B, 97 (1975).

[23] V. Dmitrašinović, Phys. Rev. D 67, 114007 (2003).

[24] L. Maiani et al., Phys. Rev. Lett. 93, 212002 (2004).

[25] C. Alexandrou, Ph. de Forcrand, and B. Lucini, Phys. Rev. Lett. 97, 222002 (2006).

[26] J. Vijande, A. Valcarce, and J.-M. Richard, arXiv:0707.3996 [PRD (to be published)]. 